\title{
Genome-wide Identification and analysis of the stress-resistance function of the TPS (Trehalose-6-Phosphate Synthase) gene family in cotton
}

Min $\mathrm{Mu}^{\dagger}, \mathrm{Xu}-\mathrm{Ke} \mathrm{Lu}^{\dagger}$, Jun-Juan Wang, De-Long Wang, Zu-Jun Yin, Shuai Wang, Wei-Li Fan and Wu-Wei Ye

\begin{abstract}
Background: Trehalose (a-D-glucopyranosyl a-D-glucopyranoside) is a nonreducing disaccharide and is widely distributed in bacteria, fungi, algae, plants and invertebrates. In the study, the identification of trehalose-6phosphate synthase (TPS) genes stress-related in cotton, and the genetic structure analysis and molecular evolution analysis of TPSs were conducted with bioinformatics methods, which could lay a foundation for further research of TPS functions in cotton.

Results: The genome information of Gossypium raimondii (group D), G. arboreum L. (group A), and G. hirsutum L. (group AD) was used in the study. Fifty-three TPSs were identified comprising 15 genes in group D, 14 in group A, and 24 in group AD. Bioinformatics methods were used to analyze the genetic structure and molecular evolution of TPS. Real-time PCR analysis was performed to investigate the expression patterns of gene family members. All TPS family members in cotton can be divided into two subfamilies: Class I and Class II. The similarity of the TPS sequence is high within the same species and close within their family relatives. The genetic structures of two TPS subfamily members are different, with more introns and a more complicated gene structure in Class I. There is a TPS domain(Glyco transf_20) at the N-terminal in all TPS family members and a TPP domain(Trehalose_PPase) at the C-terminal in all except GrTPS6, GhTPS4, and GhTPS9. All Class II members contain a UDP-forming domain. The responses to environmental stresses showed that stresses could induce the expression of TPSs but the expression patterns vary with different stresses.
\end{abstract}

Conclusions: The distribution of TPSs varies with different species but is relatively uniform on chromosomes. Genetic structure varies with different gene members, and expression levels vary with different stresses and exhibit tissue specificity. The upregulated genes in upland cotton TM-1 is significantly more than that in G. raimondii and G. arboreum L. Shixiya 1.

Keywords: TRehalose-6-phosphase synthase (TPS), Gossypium raimondii, Gossypium arboretum L, Gossypium hirsutum L, Gene family

\footnotetext{
* Correspondence: yew158@163.com

†'Equal contributors

State Key Laboratory of Cotton Biology / Institute of Cotton Research of

Chinese Academy of Agricultural Sciences, Anyang, Henan 455000, China
} 


\section{Background}

Trehalose, a non-reducing disaccharide, is composed of two glucose molecules that are connected by $\alpha, \alpha-1,1-$ glycosidic linkage and exist in bacteria, fungi, algae, invertebrates, and plants [1]. Trehalose protects bioactive substances and cell structures, such as proteins, nucleic acids, and biological membranes, under adverse environmental stresses, such as drought, freezing, oxidation, high salt, high temperature and low temperature [1-4]. Trehalose synthesis in plants is a two-step process: first, trehalose-6-phosphase synthase (TPS) catalyzes UDP-glucose and glucose-6-phosphate to generate trehalose-6-phosphate (T6P); second, trehalose-6phosphate phosphatase (TPP) catalyzes the dephosphorylation of trehalose-6-phosphate to trehalose (Fig. 1). The structure of TPS proteins in plants contains two domains: TPS and TPP; however, many studies have shown that the TPP domain in TPS proteins appears to have lost enzymatic activity during evolution $[5,6]$.

Blaquez et al. [7] screened the Arabidopsis cDNA library and, for the first time, a TPS gene was cloned named as AtTPS1 which had the trehalose-6-phosphase synthetase function from higher plants [7]. The AtTPS1 mutant TPS1 was a recessive embryonic lethal gene [8]. Even so, AtTPS1 played an important role in the process of vegetative growth and transition to flowering $[9,10]$. Zentella et al. [11] cloned TPS from Selaginella, named SITPS1, and the study found that SITPS1 maintained the biosynthesis of trehalose and played an important role in responding to heat and salt stress [11]. Studies showed that TPS expression levels in cotton increased under drought stress [12] and TPS genes in maize were also found upregulated in response to both salt and temperature stress [13]. OsTPS1 might enhance the abiotic stress tolerance of rice by increasing the trehalose and proline content [14]. Many studies have suggested that TPSs play a vital role in plants adjusting to environmental stresses.

Higher plants comprise a series of TPS gene families [15]. The Arabidopsis TPS gene family contains 11 members(AtTPS1-11) [5], while rice contains 11 members (OsTPS1-11) [16], poplar contains 12 members (PtTPS112) [6], and 28 genes for TPSs are found from Pigeon pea [17]. However, cotton TPS has not yet been systematically researched.

Cotton is an important economic and oil crop, a model plant for the study of plant polyploidy, cell wall biosynthesis, and cell elongation [18]. Cultivated cottons include both diploid and tetraploid species. The diploid cotton Gossypium raimondii D5 (group D) and the G. arboreum L. A2-8 (group A) and the tetraploid cotton G. hirsutum L. TM-1 were sequenced over the years [19-21]. This study investigated the distribution of TPSs from whole genome-wide and genetic structure of TPS genes in three cotton genomes, and examined the expression patterns of the gene family members in different tissues under different stresses. And the results were important for the study of stress-resistance mechanism and the improvement of adversity-resistance in cotton.

\section{Results \\ Genome-wide identification of cotton TPS family members}

With the GaTPS1 sequence (Accession No.: EU750912.1) and AtTPS1 sequence (Accession No.: XM_002889154.1) as references, local BLAST analysis was performed based on genomes data of G. raimondii, G. arboreum L., and upland cotton TM-1. Fourteen TPSs were found in group A, 13 genes of which were named GaTPS2-GaTPS14 according to their sequence in the chromosome; GaTPS1 was not included. Fifteen TPSs were found in group D and named GrTPS1-GrTPS15 according to their sequence in the chromosome. 24 TPSs in group AD were named GhTPS1-GhTPS24, respectively (Table 1). TPS contained from 98 to 1109 amino acid residue numbers (AA), but most contained between 800 and 1000. GhTPS4 contained 544 amino acids; GhTPS9 contained only 98. This might be associated with gene domain differences. The isoelectric point (PI) ranged from 4.59 to 8.04 . The protein molecular weight ranged from 10.76 to $130.28 \mathrm{kDa}$. The subcellular localization prediction showed that most TPSs were located in cytoplasm but some of which were located on the cytoplasmic membrane and in the nucleus.

\section{Multiple sequence alignment and phylogenetic analysis of TPS in cotton}

To assess the TPS evolutionary relationship of G. raimondii, G. arboreum L., and G. hirsutum L., multiple sequence alignment of 53 TPS family members was conducted (Additional file 1) and the evolutionary tree was constructed (Fig. 2a). According to the evolutionary tree, the cotton TPS family members were divided into two subfamilies, Class I and Class II. As shown in Fig. 2a, Class I contains 20 members, comprising 6 in G. raimondii group D, 5 in G. arboreum L. group A, and 9 in

\begin{tabular}{|c|c|c|c|c|}
\hline \multirow{2}{*}{$\begin{array}{c}\text { UDP-glucose } \\
+ \\
\text { Glucose-6-phosphate }\end{array}$} & $\begin{array}{c}\text { Trehalose-6-phosphase } \\
\text { synthase }\end{array}$ & \multirow{2}{*}{ Trehalose-6-phosphate } & $\begin{array}{c}\text { Trehalose-6-phosphate } \\
\text { phosphatase }\end{array}$ & \multirow{2}{*}{ Trehalose } \\
\hline & ( TPS ) & & ( TPP) & \\
\hline
\end{tabular}


Table 1 Basic characteristic of TPS genes in cotton genome

\begin{tabular}{|c|c|c|c|c|c|c|}
\hline $\begin{array}{l}\text { Gene } \\
\text { name }\end{array}$ & Accession number & $\begin{array}{l}\text { CDS } \\
\text { (bp) }\end{array}$ & $\mathrm{AA}$ & $\mathrm{pl}$ & $\begin{array}{l}\mathrm{Mw} \\
(\mathrm{kDa})\end{array}$ & $\begin{array}{l}\text { Predicted } \\
\text { subcellular } \\
\text { localization }\end{array}$ \\
\hline GaTPS1 & EU750912.1 & 2586 & 861 & 6.27 & 96.96 & Cytoplasmic \\
\hline GaTPS2 & Cotton_A_09724 & 2589 & 862 & 5.74 & 97.6 & Cytoplasmic \\
\hline GaTPS3 & Cotton_A_38312 & 2517 & 838 & 5.74 & 95.55 & Cytoplasmic \\
\hline GaTPS4 & Cotton_A_22808 & 2610 & 869 & 5.75 & 98.49 & Nuclear \\
\hline GaTPS5 & Cotton_A_05024 & 2574 & 857 & 5.94 & 97.22 & Cytoplasmic \\
\hline GaTPS6 & Cotton_A_31871 & 2829 & 942 & 6.91 & 106.42 & Cytoplasmic \\
\hline GaTPS7 & Cotton_A_22769 & 2829 & 942 & 6.43 & 106.17 & Cytoplasmic \\
\hline GaTPS8 & Cotton_A_02720 & 2562 & 853 & 5.63 & 96.39 & Plasma Membrane \\
\hline GaTPS9 & Cotton_A_17512 & 2574 & 857 & 5.78 & 96.64 & Cytoplasmic \\
\hline GaTPS10 & Cotton_A_12395 & 2574 & 857 & 5.70 & 96.88 & Nuclear \\
\hline GaTPS11 & Cotton_A_12382 & 2952 & 983 & 6.33 & 111.4 & Cytoplasmic \\
\hline GaTPS12 & Cotton_A_23712 & 2754 & 917 & 5.66 & 104.05 & Cytoplasmic \\
\hline GaTPS13 & Cotton_A_23709 & 2652 & 883 & 6.58 & 99.37 & Cytoplasmic \\
\hline GaTPS14 & Cotton_A_09181 & 2595 & 864 & 5.75 & 97.4 & Cytoplasmic \\
\hline GrTPS1 & Cotton_D_gene_10022693 & 2562 & 853 & 5.84 & 96.33 & Plasma Membrane \\
\hline GrTPS2 & Cotton_D_gene_10020837 & 2808 & 935 & 6.70 & 105.47 & Cytoplasmic \\
\hline GrTPS3 & Cotton_D_gene_10038561 & 2517 & 848 & 5.74 & 95.54 & Cytoplasmic \\
\hline GrTPS4 & Cotton_D_gene_10020702 & 2754 & 917 & 5.67 & 104.01 & Cytoplasmic \\
\hline GrTPS5 & Cotton_D_gene_10020699 & 3507 & 1168 & 6.39 & 130.28 & Plasma Membrane \\
\hline GrTPS6 & Cotton_D_gene_10020701 & 2517 & 838 & 8.04 & 93.69 & Plasma Membrane \\
\hline GrTPS7 & Cotton_D_gene_10033185 & 2595 & 864 & 5.77 & 97.6 & Cytoplasmic \\
\hline GrTPS8 & Cotton_D_gene_10037806 & 2589 & 862 & 5.8 & 97.67 & Cytoplasmic \\
\hline GrTPS9 & Cotton_D_gene_10033657 & 2574 & 857 & 5.66 & 96.84 & Cytoplasmic \\
\hline GrTPS10 & Cotton_D_gene_10033644 & 2886 & 961 & 6.27 & 108.94 & Cytoplasmic \\
\hline GrTPS11 & Cotton_D_gene_10000478 & 2517 & 838 & 5.89 & 94.92 & Cytoplasmic \\
\hline GrTPS12 & Cotton_D_gene_10031457 & 2574 & 857 & 5.7 & 96.53 & Cytoplasmic \\
\hline GrTPS13 & Cotton_D_gene_10023754 & 2610 & 869 & 5.7 & 98.48 & Nuclear \\
\hline GrTPS14 & Cotton_D_gene_10006852 & 2829 & 942 & 6.21 & 105.94 & Cytoplasmic \\
\hline GrTPS15 & Cotton_D_gene_10009065 & 2586 & 861 & 6.24 & 96.88 & Cytoplasmic \\
\hline GhTPS1 & CotAD_09030 & 2673 & 890 & 5.9 & 100.86 & Plasma Membrane \\
\hline GhTPS2 & CotAD_24641 & 2610 & 869 & 5.7 & 98.489 & Nuclear \\
\hline GhTPS3 & CotAD_51660 & 2574 & 857 & 5.85 & 96.64 & Cytoplasmic \\
\hline GhTPS4 & CotAD_66147 & 1635 & 544 & 6.39 & 61.95 & Cytoplasmic \\
\hline GhTPS5 & CotAD_22604 & 2826 & 941 & 5.71 & 106.63 & Cytoplasmic \\
\hline GhTPS6 & CotAD_22606 & 2652 & 883 & 6.41 & 99.349 & Cytoplasmic \\
\hline GhTPS7 & CotAD_03946 & 2595 & 864 & 5.82 & 97.57 & Cytoplasmic \\
\hline GhTPS8 & CotAD_16380 & 2634 & 877 & 6.42 & 98.72 & Cytoplasmic \\
\hline GhTPS9 & CotAD_16379 & 297 & 98 & 4.59 & 10.76 & Extracellular \\
\hline GhTPS10 & CotAD_25711 & 2574 & 857 & 5.61 & 96.84 & Nuclear \\
\hline GhTPS11 & CotAD_21592 & 2829 & 942 & 6.3 & 106 & Cytoplasmic \\
\hline GhTPS12 & CotAD_25696 & 2808 & 935 & 6.83 & 106.28 & Nuclear \\
\hline GhTPS13 & CotAD_66378 & 2517 & 838 & 5.7 & 95.58 & Cytoplasmic \\
\hline GhTPS14 & CotAD_16567 & 2589 & 862 & 5.7 & 97.66 & Cytoplasmic \\
\hline
\end{tabular}


Table 1 Basic characteristic of TPS genes in cotton genome (Continued)

\begin{tabular}{lllllll}
\hline GhTPS15 & CotAD_52287 & 2952 & 983 & 6.27 & 111.48 & Cytoplasmic \\
GhTPS16 & CotAD_53302 & 2286 & 761 & 5.75 & 86.67 & Cytoplasmic \\
GhTPS17 & CotAD_74149 & 2559 & 755 & 6.27 & 85.75 & Cytoplasmic \\
GhTPS18 & CotAD_51342 & 2574 & 857 & 5.7 & 96.91 & Nuclear \\
GhTPS19 & CotAD_05656 & 2574 & 857 & 5.86 & 96.97 & Cytoplasmic \\
GhTPS20 & CotAD_00585 & 2574 & 857 & 5.78 & 96.57 & Cytoplasmic \\
GhTPS21 & CotAD_17819 & 2562 & 853 & 5.67 & 96.34 & Plasma Membrane \\
GhTPS22 & CotAD_24751 & 2808 & 935 & 6.7 & 105.44 & Cytoplasmic \\
GhTPS23 & CotAD_36474 & 2589 & 862 & 5.85 & 97.73 & Cytoplasmic \\
GhTPS24 & CotAD_40190 & 2808 & 935 & 6.8 & 105.57 & Cytoplasmic \\
\hline
\end{tabular}

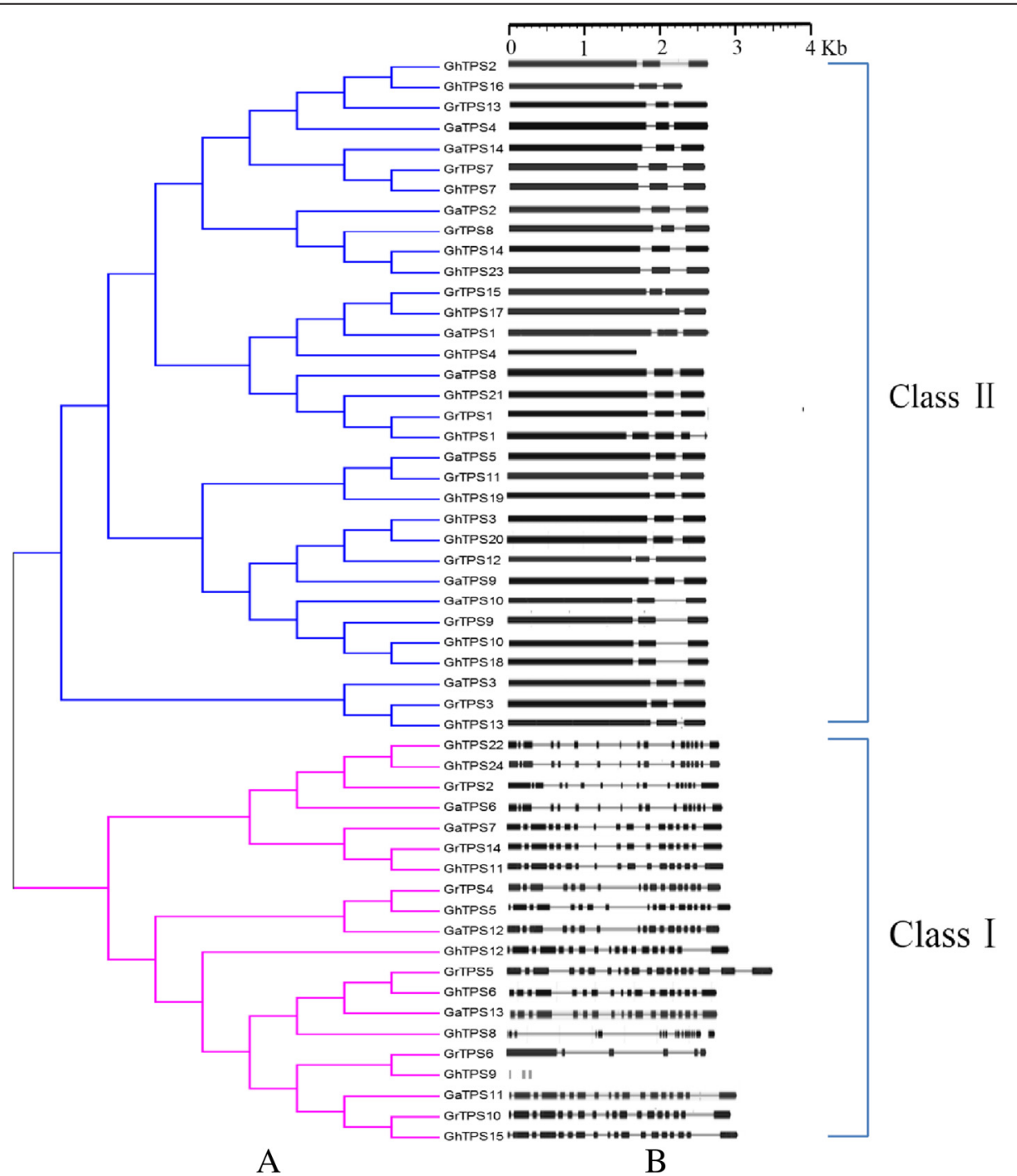

Fig. 2 Phylogenetic analysis (a) and gene structure (b) of the TPS gene family in cotton. The cotton TPS family members were divided into two subfamilies, Class I and Class II. Exons —Introns 
upland cotton group AD. Class II contains 33 members, comprising 9, 9, and 15 members in groups $\mathrm{D}, \mathrm{A}$, and $\mathrm{AD}$, respectively.

\section{Gene structures and protein domains of cotton TPSs}

Gene structure analysis is an important method by which to study genetic evolution. The numbers of introns and exons in TPS family members were calculated in G. raimondii, G. arboreum L., and G. hirsutum L. and the TPS structure in cotton was created (Fig. 2b). The result showed that, except for GrTPS5, GhTPS4, and GhTPS9, the code length of the remaining 50 family members ranged from
2500 to $3000 \mathrm{bp}$. Not any big difference occurred in gene length; however, the difference in the genetic structure of TPSs was significant between the two subfamily members, where the number of gene introns was larger and the genetic structures were more complicated in Class I than in Class II.

The domain analysis showed that, with the exception of GrTPS6, GhTPS4, and GhTPS9, a TPS structure domain (Glyco_transf_20) in 53 cotton TPS family members is located at the N-terminal and a TPP domain (Trehalose_PPase) at the C-terminal. GrTPS6, GhTPS4, and GhTPS9 contain only the TPS domain.

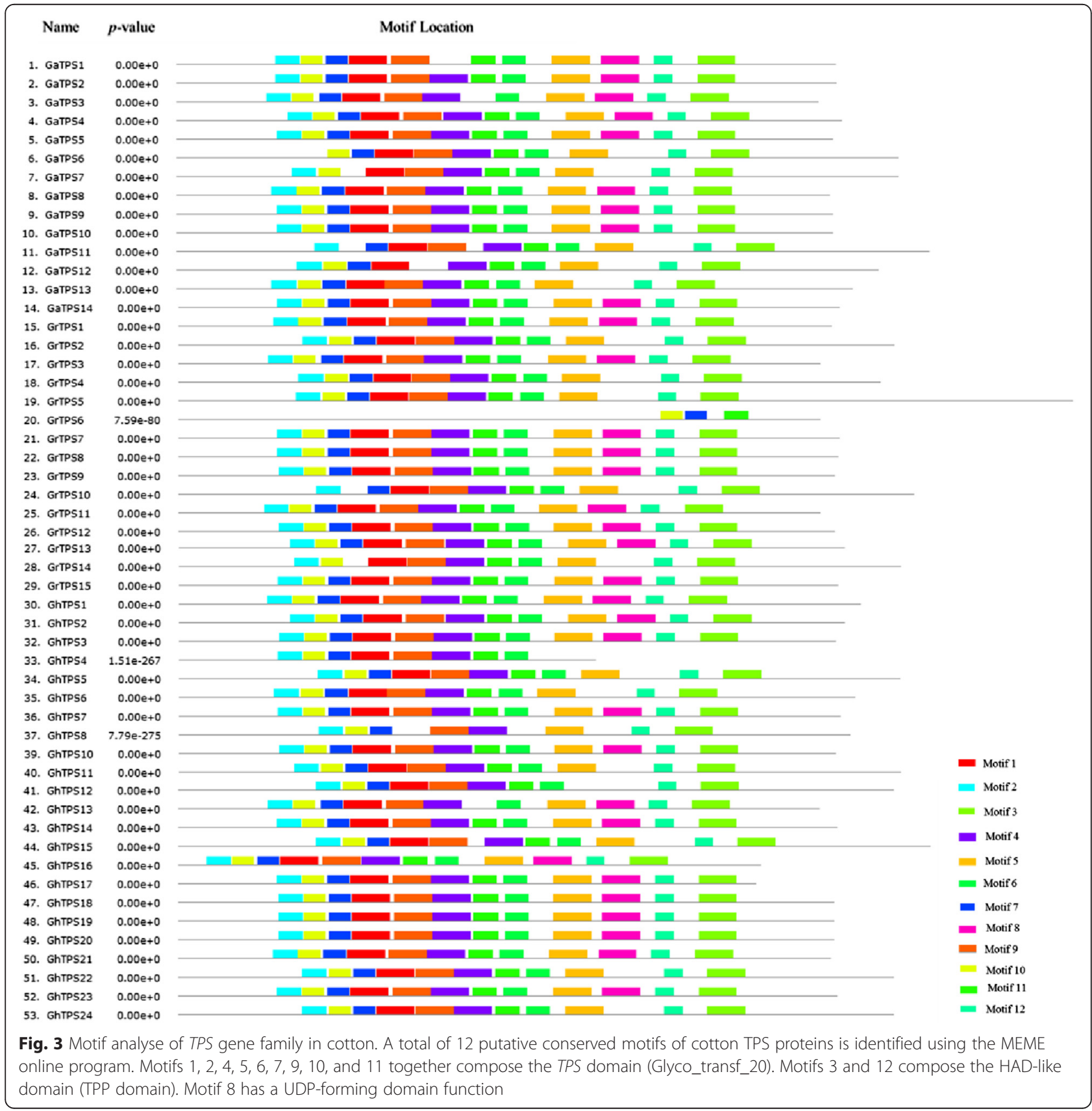


Motif analysis of 53 family members is shown in Fig. 3. Cotton TPSs totally contain 12 motifs (Additional file 2: Figure S1). Among them, motifs 1, 2, 4, 5, 6, 7, 9, 10, and 11 together compose the TPS domain (Glyco_transf_20). Motifs 3 and 12 compose the HAD-like domain (TPP domain). But motif 8 has a UDP-forming domain that functions separately. It was found that motifs $1,3,5,6$, and 12 in group D are conservative, while only motif 11 is conservative in group A. Motif 2 was observed in all group AD members. GrTPS6 contains an incomplete TPS domain: only motifs 7,10 , and 11 . GhTPS4 contains all TPS domains except for motif 5 . GhTPS9 contains only incomplete motif 2, which is not listed in the Fig. 3. It was speculated that this might be caused in the long evolutionary process. In addition, 20 among the 53 genes without motif 8 are in perfect accord with Class I members in the evolutionary tree (Fig. 2a).

\section{Distribution and duplication analysis of TPS family members}

Gene distribution on the chromosome could provide an important basis for the study of the genes evolution and functions. Combined with the chromosome information on three cotton reference genomes and the TPS locations, the cotton TPSs distribution map on chromosomes can be

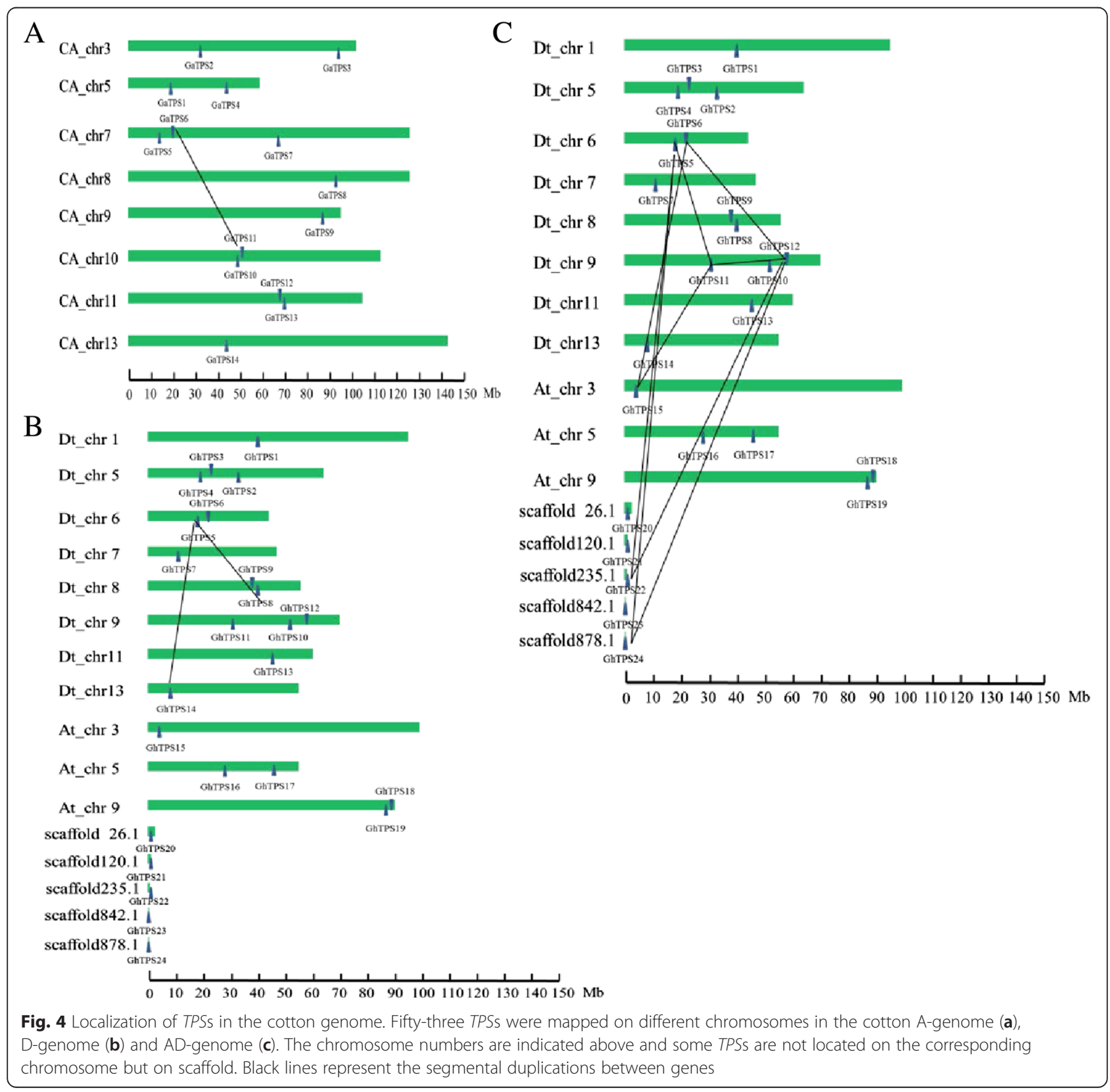


drawn (Fig. 4). G. arboreum L. has 14 GaTPSs, which are located on total 8 chromosomes. There are two genes on chromosomes $3,5,10$, and 11 , separately and only one gene is located on chromosome 8, 9, and 13, separately. However, the rest of three genes are located on chromosome 7 (Fig. 4a). Among the 15 GrTPSs from G. raimondii, three are located on chromosome 6, two on chromosome 9, and one each on chromosomes $1,2,4,7,8,10,11,13$. The remaining two genes are not located on a chromosome-GrTPS14 is located on scaffold 254 and GrTPS15 on scaffold 321 (Fig. 4b). Five TPSs in upland cotton are not located on the corresponding chromosome but on scaffold 26.1, 120.1, 235.1, 842.1, and scaffold 878.1, respectively. The remaining 19 genes from subgroups $A$ and D are unevenly distributed. In subgroup A, two genes are located on chromosome 5, two on chromosome 9, and 1 on chromosome 3 . In subgroup $\mathrm{D}$, three genes are located on chromosomes 5 and 9 separately, two on chromosomes 6 and 8 , and each of the remaining genes on chromosomes 1 , 7, 11, and 13, respectively (Fig. 4c).

Gene duplications in genomes could provide important information for gene evolution analysis. In the research, we performed gene duplication analysis in genome $\mathrm{A}$, genome $\mathrm{D}$ and genome $\mathrm{AD}$, respectively (Fig. 4). Usually, the criteria for inferring a gene duplication event are (1) the length of the alignment sequence covers $\geq 75 \%$ of the longest gene, and (2) the similarity of the aligned regions is $\geq 70 \%[22,23]$. We totally found 12 gene pairs may be associated with gene duplications. One is in genome A (GaTPS6/11), and one is in genome $\mathrm{D}($ GrTPS5/14) and others are in genome AD. This characteristic in the same genome is important for gene divergence. And the similar gene structure and gene functions in each gene family may be the result of gene expansion from ancient paralogs or multiple origins of gene ancestry [24]. It was reported that partial fragment replication of the chromosome regions might lead to scattered distribution of gene family members on several chromosomes [25]. Compared with other eukaryotes, plants have a higher rate of gene replication [26] and this might cause an uneven distribution of TPS family members. It was reported that whole genome replication occurs in G. raimondii at least twice. Currently, 2355 linear modules and 39 triple replication regions have been identified [19] and gene duplication and postseparation phenomenon are the two main driving forces of evolution [27, 28].

\section{Cotton TPS family relationships with other plant TPSs}

A phylogenetic tree was used to reveal homologous relationships and evolutionary roots of TPS from different species. To reveal the evolutionary relationship between the cotton TPS family members and those from Arabidopsis, rice, Zea mays, and soybeans, the amino acid sequence alignment of all members from those species was conducted. MEGA 5.1 was used to construct the phylogenetic tree (Fig. 5). The results showed that compared with the other four species, the relative coefficient of TPS from three cotton genomes is higher, indicating a closer relationship. GrTPS2 and GrTPS14 of G. raimondii, GaTPS6 and GaTPS7 of G. arboreum L., and GhTPS11, GhTPS22, and GhTPS24 of upland cotton have a closer relationship with TPS1 of Arabidopsis, which suggests that they have similar functions. In addition, parts of the cotton and soybean TPS family members are grouped together and show relatively close evolutionary relationships.

\section{Cotton TPS expression pattern analysis under different stresses}

To study the expression patterns of TPS family members in different tissues under low temperature, drought, and salt stress, G. raimondii, G. arboreum L. Shixiya 1, and G. hirsutum L. TM-1 were cultivated at trefoil stage. Real-time quantification PCR after different stresses was conducted. The results showed that most of TPS family members expressed in three tissues, including roots, stems, and leaves, and the gene expression levels changed after treatments (Fig. 6). After being exposed to $4.0{ }^{\circ} \mathrm{C}$ for $24 \mathrm{~h}, 6$ genes in the roots were upregulated and 8 genes were downregulated in G. arboreum L., 5 genes upregulated and 7 genes downregulated in G. raimondii, and 19 genes upregulated in upland cotton. 9, 7, and 17 genes in stem were upregulated in groups $A, D$, and $A D$, respectively. 12 genes in leaves upregulated in group A, 8 upregulated in group D, and 10 upregulated while 12 downregulated in group AD.

After exposure to $150 \mathrm{mM} \mathrm{NaCl}, 8,8$, and10 genes in roots upregulated in the three genome groups, respectively. In stems, 7 genes upregulated and 7 downregulated in group A, 4 upregulated and 11 downregulated in group D, and 13 upregulated and 11 downregulated in group $\mathrm{AD}$. In leaves, three and four genes downregulated in group $\mathrm{D}$ and group $\mathrm{AD}$, respectively, and seven downregulated in group A.

After being exposed to drought stress, 7 genes in roots upregulated and 7 downregulated in group A, 8 upregulated and 7 downregulated in group D, and 11 upregulated and 13 genes downregulated in group AD. In stems, 7 genes upregulated significantly in group A, only the GrTPS5 expression level increased in group D, and the expression level of 10 genes increased in group AD. In leaves, 11, 7, and 16 genes upregulated expressed in the 3 groups, respectively.

After exposure to low temperature, salt, or drought, the expression level of GrTPS12 in group D remained constant in the roots, stems, and leaves. The remaining genes were downregulated (Fig. 6). The expression level 


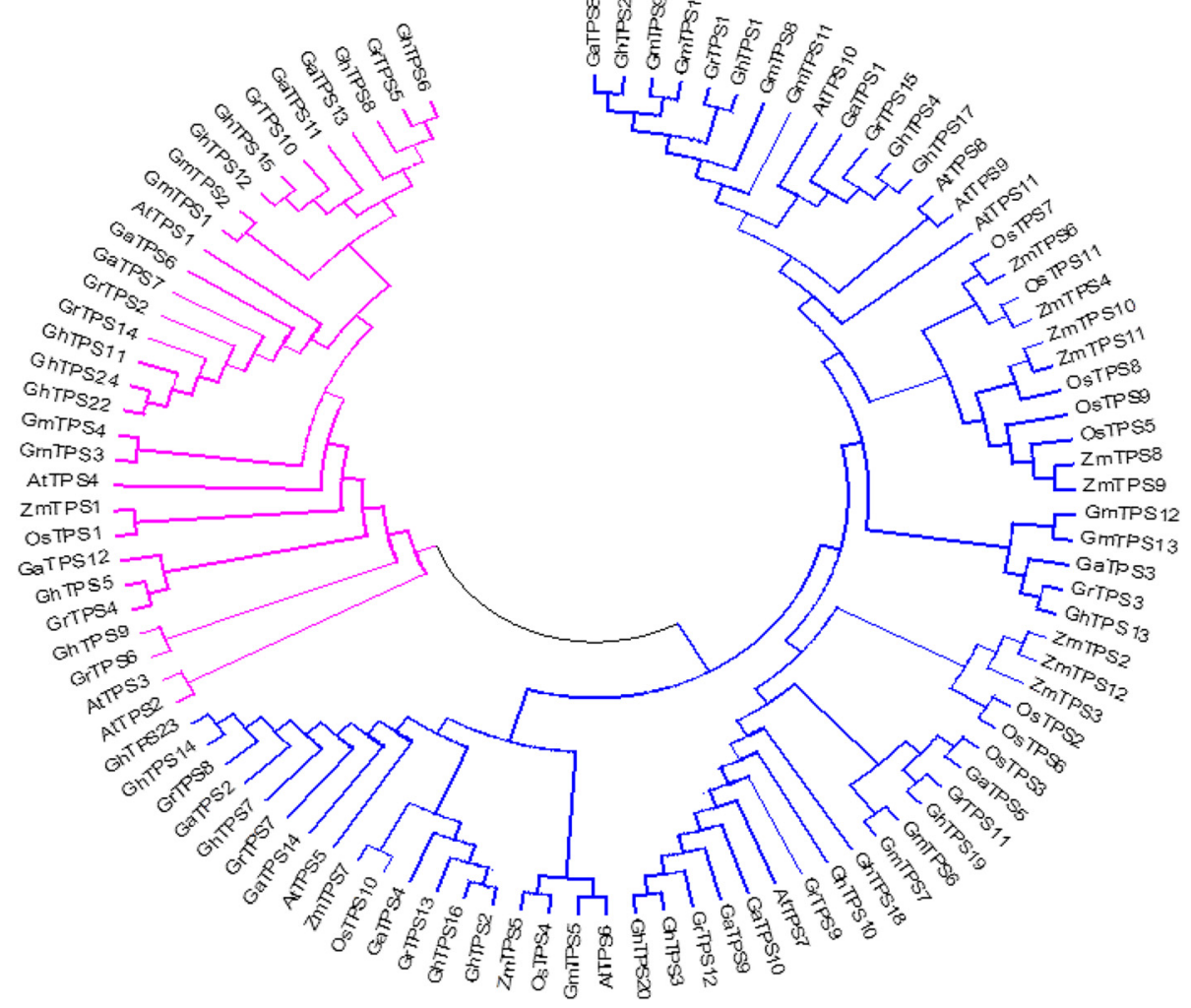

Fig. 5 Phylogenetic analysis of the TPS gene family in cotton and other plants. The joint unrooted phylogenetic tree containing 14 G. raimondii (GrTPS), 13 G. arboreum L. (GaTPS), 24 G. hirsutum L. (GhTPS), 11 Arabidopsis (AtTPS), 11 rice (OsTPS), 13 soybeans (GmTPS) and 12 Zea mays(ZmTPS) TPS genes was constructed using neighbor-joining methods

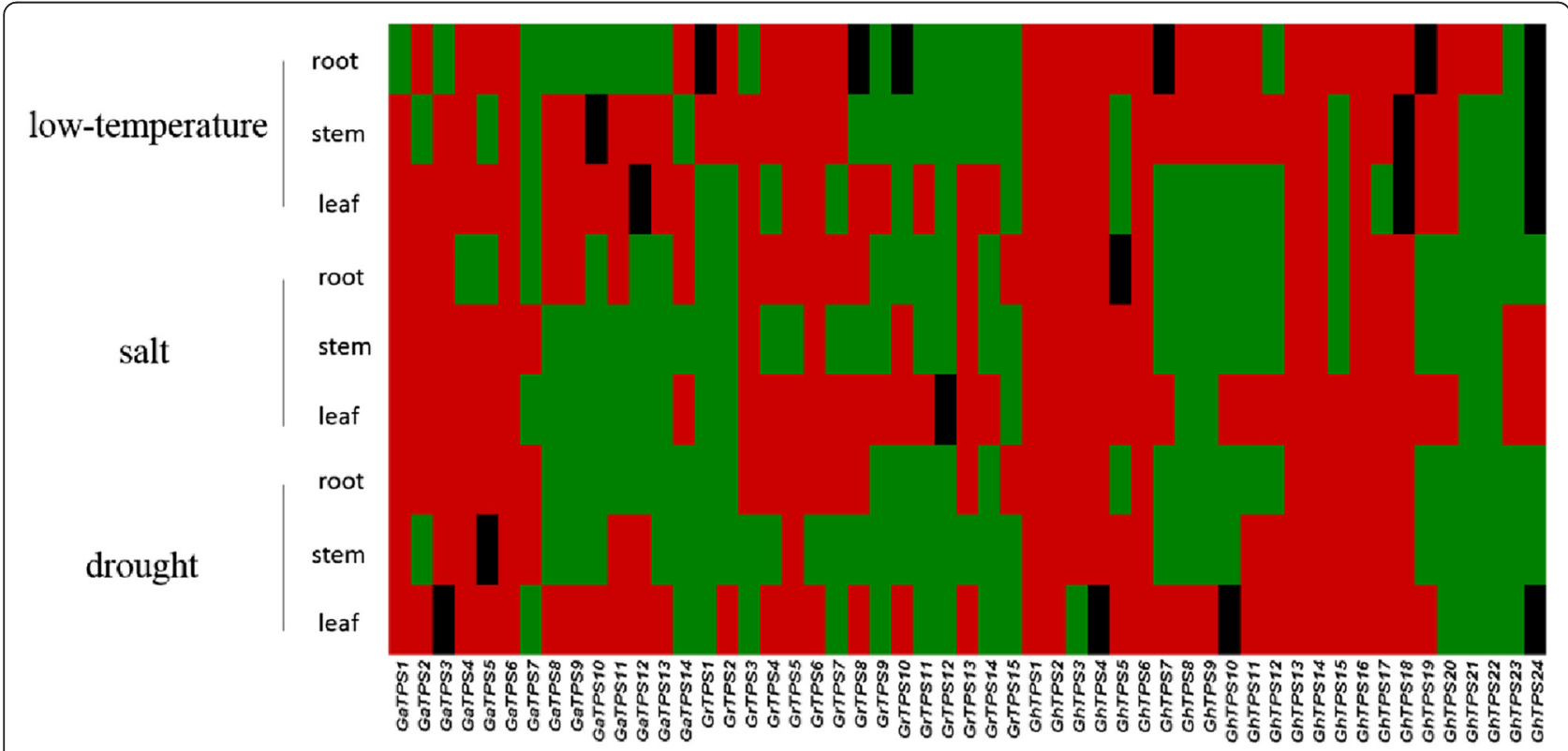

Fig. 6 Expression patterns of TPS gene family under conditions of cold, salt and drought stress. Quantitative RT-PCR analysis of the expression level of TPSs in G. raimondii, G. arboreum L. Shixiya 1, and G. hirsutum L. TM-1 including roots, stems and leaves. Results were normalized using Histone-3 (Accession No. AF02471) as the internal control. Red represents genes upregulated; black represents genes which had no significant change in expression; green represents genes downregulated 
of GaTPS6 in group A and GhTPS1, GhTPS2, GhTPS6, GhTPS13, GhTPS14, and GhTPS16 in group AD significantly increased. It was speculated that these genes in $G$. arboreum L., Shixiya 1 and upland cotton TM-1 played important roles in stress tolerance.

\section{Discussion}

With the increasing research in genomes, comparative genomics methods are used to study gene families, which is one of the hot research topics for several species. Many gene families in different species were identified, such as soybean $L E A$ [29], tomato $L B D$ [30], MAPK [31], and cotton MAPKKK [32]. Cotton was one of the pioneer plants in the saline-alkali. TPS is closely related to stress resistance and the study of TPS from the whole genome would be very important for cotton breeding and the research of stress-resistance mechanism in cotton.

This research investigated G. raimondii, G. arboreum L., and the upland cotton with the GaTPS1 sequence (Gene Bank No.: EU750912.1) and AtTPS1 sequence (Accession No.: XM_002889154.1) as the reference sequences. And totally 53 family members were identified including 15 in group D, 14 in group A, and 24 in group $\mathrm{AD}$, which indicated TPS genes in AD genome were more than that in both A genome and $\mathrm{D}$ genome, but not the sum of two genomes. This may be associated with the gene duplications in the evolution of AD genome from their Diploid ancestors. Gene duplication analysis showed 12 gene pairs probably were linked with gene duplication, which was important for their functions research.

Cotton TPSs can be divided into two families-20 genes in Class I and 33 genes in Class II, which was consistent with previous studies in Arabidopsis, rice, and Populus [16]. Domains analysis showed that there is a TPS domain (Glycotransf_20) in all cotton TPS members at the $\mathrm{N}$-terminal, while a TPP domain (Trehalose_PPase) at the $\mathrm{C}$ - terminal in most TPS genes. Functional domains analysis suggested that these domains might be important for TPS functions. The process and specific function of each domain involved in regulating function and metabolic pathways remains to be determined with additional studies.

Trehalose could protect bioactive substances and cell structures, such as proteins, nucleic acids, and biological membranes, under adverse environmental stresses, such as high salt, drought, high temperature, freezing, and oxidation [1-4]. In this study, after exposing G. raimondii, G. arboreum L., Shixiya 1, and upland cotton TM-1 to low temperature $\left(4.0{ }^{\circ} \mathrm{C}\right)$, salt $(150 \mathrm{mM} \mathrm{NaCl})$, and drought stress (sand moisture content $5.0 \%$ ), it was found that, except for GaTPS6 in group A and GhTPS1, GhTPS2, GhTPS6, GhTPS13, GhTPS14, and GhTPS16 in group $\mathrm{AD}$, the expression levels of the remaining 46 family members varied greatly in different tissues. In group A, GaTPS3 expression levels in stems and leaves increased after exposure to low temperature and salt, but decreased in roots after exposure to low temperature and remained constant in leaves after exposure to drought. In group D, the expression level of GaTPS14 in leaves increased after exposure to low temperature and salt, while that of other genes decreased. In group AD, the expression level of GaTPS18 didn't change obviously in stems and roots after exposure to low temperatures but increased in each tissue after other stress treatments. The expression level of GaTPS22 decreased in different tissues after stress treatments, with the exception of increased in roots after exposure to low temperatures. It was found that the rates of upregulated genes in different tissues in upland cotton TM-1 were mostly more than that in G. raimondii and G. arboreum L. Shixiya 1, which also may be associated the stronger tolerance of upland cotton TM-1 to various stresses compared with two diploid cottons.

TPS has been found in many plants [5, 6, 12, 13, 33]. The drought tolerance of Arabidopsis with overexpressed AtTPS1 has significantly improved [34]. OsTPS1 could improve rice-seed tolerance to low temperature, salt, and drought [14]. In this study, the expression levels of GrTPS5 and GhTPS15 in different tissues increased under drought stress, which agrees with the results of studies on Arabidopsis. GrTPS5 expression levels increased under low temperature stress. GhTPS15 expression levels also increased in roots under low temperature stress and in leaves under salt stress. GaTPS6 and GhTPS6 expression levels increased under low temperature, salt, and drought stress, which was in accord with that of rice OsTPS1. Previous studies showed that overexpression of AtTPS1 and OsTPS1 in Arabidopsis and rice caused phenotypic changes, plant height reduction, and late blooming $[9,10]$. It was speculated that overexpression of GrTPS5, GaTPS6, GhTPS6, and GhTPS15 in cotton also caused the same phenotypic changes. In addition, it was speculated that the four genes played important roles in cotton against stresses according to their expression levels under stress conditions. Previous reports have shown that GaTPS1 is closely related to drought resistance of G. arboreum L. [12]. In this study, the expression level of GaTPS1 increased significantly in roots, stems, and leaves under drought stress. And also increased in stems and leaves under low temperature stress.

\section{Conclusions}

In this study, totally 53 TPS genes in total were identified, including 15, 14 and 24 in group D, group A and group $\mathrm{AD}$, respectively. All TPS gene members except 
GrTPS6, GhTPS4, and GhTPS9, contain a TPS domain(Glyco transf_20) at the N-terminal in TPS family members and a TPP domain(Trehalose_PPase) at the Cterminal. Most TPS genes could be induced by different stresses, including drought, salt and low temperature, revealing that TPS genes may play a vital role in response to stresses. The study lays a foundation for the study of TPS functions and the research of cotton growth and development.

\section{Methods}

\section{Identification of cotton TPS family members}

The local BLAST was conducted with the GaTPS1 (Gene Bank No. : EU750912.1) sequence and AtTPS1 gene sequence (Gene Bank No. : XM_002889154.1) as reference genes in diploid cotton G. raimondii (DD) and G. arboreum L.(AA) and a tetraploid cotton G. hirsutum L. (AADD) genomes. All genomes data of cotton were derived from the Institute of Cotton Research, Chinese Academy of Agricultural Sciences, Anyang, China. Evalue $=0.0001$ was set to predict the cotton TPS family. The screened protein sequences were further confirmed according to their conserved domains using the online conserved domains analytical tool (http://www.ncbi.nlm.nih.gov/Structure/cdd/wrpsb.cgi).

\section{Phylogenetic analysis}

The data of TPS protein sequences of Arabidopsis were downloaded from the Arabidopsis genome database (https://www.arabidopsis.org/). Rice TPS protein sequences were downloaded from the rice genome database (http://rice.plantbiology.msu.edu/). Glycine max (L.) Merrill TPS protein sequences were downloaded from Phytozome 11.0 (https://phytozome.jgi.doe.gov/pz/ portal.html). Zea mays TPS protein sequences were downloaded from NCBI (http://www.ncbi.nlm.nih.gov/) and Phytozome 11.0 (https://phytozome.jgi.doe.gov/pz/ portal.html/). TPS in Arabidopsis, rice, soybeans and Zea mays was named AtTPS, OsTPS, GmTPS, and ZmTPS, respectively. Multiple alignment of TPS proteins from the diploid cotton G. raimondii and G. arboreum L. and a tetraploid cotton G. hirsutum L. was performed using MEGA 5.1 [35]. Neighbor-joining method was used to construct gene trees and structure diagram.

\section{Basic structure of TPS gene family}

ProParam online tool in ExPASy (http://www.expasy.org/ tools/protparam.html) was used to analyze the basic physical and chemical properties of the protein sequences. Subcellular localization predictor (http://cello.life.nctu.edu.tw/) was used to predict subcellular localizations. The domain of the TPSs was analyzed using the conserved domains searcher tool (http://www.ncbi.nlm.nih.gov/Structure/cdd/ wrpsb.cgi), and the motif analysis was carried out using MEME program (http://meme-suite.org/).

Expression patterns analysis of cotton TPSs under stresses The test cottons G. raimondii, G. arboreum L. Shixiya 1, and G. hirsutum L. TM-1were provided from the Institute of Cotton Research, Chinese Academy of Agricultural Sciences (CAAS, Anyang, China). Cotton plants were cultivated using the sand culture method [36]. Three seedlings at trefoil stage were exposed to low temperature $\left(4.0{ }^{\circ} \mathrm{C}, 24 \mathrm{~h}\right)$, salt $(150 \mathrm{mM} \mathrm{NaCl}, 24 \mathrm{~h})$ and drought (sand moisture content $5.0 \%$ ), respectively. Then the roots, stems, and leaves were sampled and frozen in liquid nitrogen at $-80{ }^{\circ} \mathrm{C}$. Total RNA was extracted and reverse transcribed into cDNA. Primer Premier 5.0 (PREMIER Biosoft) was used to design fluorescent quantitative primers (Additional file 3: Table S1) for qRT-PCR. Fluorescent quantitative real-time polymerase chain reaction (qRT-PCR) was performed using Histone-3 (Accession No. AF02471) as a reference gene. PCR reaction conditions and programs were set as follows: $94{ }^{\circ} \mathrm{C}$ for $30 \mathrm{~s}, 94{ }^{\circ} \mathrm{C}$ for $5.0 \mathrm{~s}, 55{ }^{\circ} \mathrm{C}$ for $34 \mathrm{~s}$, and $72{ }^{\circ} \mathrm{C}$ for $34 \mathrm{~s}$ for a total of 40 cycles. Relative quantitative analysis of 20 target genes was calculated with the $2^{-\Delta \Delta C T}$ method.

\section{Availability of supporting data}

The data sets supporting the results of this article are included within the article and its additional files.

\section{Additional files}

Additional file 1: Multiple alignment analysis of cotton TPSS (FAS $83 \mathrm{~kb}$ ) Additional file 2: Figure S1. Motif sequences of cotton TPSS (DOCX 999 kb)

Additional file 3: Table S1. Primers used for qRT-PCR (XLS 15 kb)

Abbreviations

GaTPSs: genes in G. arboreum L. (AA); GhTPSs: genes in Gossypium hirsutum L. (AADD); GrTPSs: genes in G. raimondii (DD); TPS: trehalose-6-phosphate synthase.

\section{Competing interests}

The authors declare that they have no competing interests.

\section{Authors' contributions}

WY conceived the experiment. MM, XL, JW participated in the design of the study and performed the statistical analysis. MM and XL drafted the manuscript. DW, ZY contributed the experiment and helped to draft the manuscript. SW and WF provided the materials and revised the manuscript. All authors have read and approved the final manuscript.

\section{Acknowledgments}

This work was supported by grants from The China Major Projects for Genetically modified organisms breeding (Grant No. 2014ZX08005-04B). We also appreciate the reviewers and editors for their patience to the work.

Received: 20 November 2015 Accepted: 7 March 2016

Published online: 18 March 2016 


\section{References}

1. Elbein AD, Pan YT, Pastuszak I, Carroll D. New insights on trehalose: a multifunctional molecule. Glycobiology. 2003;13(4):17r.

2. Garg AK, Kim JK, Owens TG, Ranwala AP, Do Choi Y, Kochian LV, Wu RJ. Trehalose accumulation in rice plants confers high tolerance levels to different abiotic stresses. Proc Natl Acad Sci U S A. 2002:99(25):15898-903.

3. Goddijn OJM, van Dun K. Trehalose metabolism in plants. Trends Plant Sci. 1999:4(8):315-9.

4. Jang IC, Oh SJ, Seo JS, Choi WB, Song SI, Kim CH, Seo HS, Do Choi Y, Nahm $\mathrm{BH}$, et al. Expression of a bifunctional fusion of the Escherichia coli genes for trehalose-6-phosphate synthase and trehalose-6-phosphate phosphatase in transgenic rice plants increases trehalose accumulation and abiotic stress tolerance without stunting growth. Plant Physiol. 2003;131(2):516-24.

5. Vandesteene L, Ramon M, Le Roy K, Van Dijck P, Rolland F. A single active trehalose-6-P synthase (TPS) and a family of putative regulatory TPS-like proteins in Arabidopsis. Mol Plant. 2010;3(2):406-19.

6. Zang BS, Li HW, Li WJ, Deng XW, Wang XP. Analysis of trehalose-6phosphate synthase (TPS) gene family suggests the formation of TPS complexes in rice. Plant Mol Biol. 2011;76(6):507-22.

7. Blazquez MA, Santos E, Flores CL, Martinez-Zapater JM, Salinas J, Gancedo C. Isolation and molecular characterization of the Arabidopsis TPS1 gene, encoding trehalose-6-phosphate synthase. Plant J. 1998;13(5):685-9.

8. Eastmond PJ, van Dijken AJH, Spielman M, Kerr A, Tissier AF, Dickinson HG, Jones JDG, Smeekens SC, Graham IA. Trehalose-6-phosphate synthase 1, which catalyses the first step in trehalose synthesis, is essential for Arabidopsis embryo maturation. Plant J. 2002;29(2):225-35.

9. van Dijken AJH, Schluepmann H, Smeekens SCM. Arabidopsis trehalose-6phosphate synthase 1 is essential for normal vegetative growth and transition to flowering. Plant Physiol. 2004;135(2):969-77.

10. Gomez LD, Baud S, Gilday A, Li Y, Graham IA. Delayed embryo development in the ARABIDOPSIS TREHALOSE-6-PHOSPHATE SYNTHASE 1 mutant is associated with altered cell wall structure, decreased cell division and starch accumulation. Plant J. 2006;46(1):69-84.

11. Zentella R, Mascorro-Gallardo JO, Van Dijck P, Folch-Mallol J, Bonini B, Van Vaeck C, Gaxiola R, Covarrubias AA, Nieto-Sotelo J, Thevelein JM et al. A Selaginella lepidophylla trehalose-6-phosphate synthase complements growth and stress-tolerance defects in a yeast tps 1 mutant. Plant Physiol. 1999:119(4):1473-82.

12. Kosmas SA, Argyrokastritis A, Loukas MG, Eliopoulos E, Tsakas S, Kaltsikes PJ. Isolation and characterization of drought-related trehalose 6-phosphatesynthase gene from cultivated cotton (Gossypium hirsutum L.). Planta. 2006; 223(2):329-39.

13. Jiang W, Fu FL, Zhang SZ, Wu L, Li WC. Cloning and characterization of functional trehalose-6-phosphate synthase gene in maize. J Plant Biol. 2010; 53(2):134-41.

14. Li HW, Zang BS, Deng XW, Wang XP. Overexpression of the trehalose-6phosphate synthase gene OSTPS1 enhances abiotic stress tolerance in rice. Planta. 2011;234(5):1007-18.

15. Lunn JE. Gene families and evolution of trehalose metabolism in plants. Funct Plant Biol. 2007;34(6):550-63.

16. Yang HL, Liu YJ, Wang CL, Zeng QY. Molecular evolution of trehalose-6phosphate synthase (TPS) gene family in Populus, Arabidopsis and rice. Plos One. 2012;7(8), e42438. doi:10.1371/journal.pone.0042438.

17. Singh NK, Gupta DK, Jayaswal PK, Mahato AK, Dutta S, Singh S, Dogra V, Singh BP, Kumawat $\mathrm{G}$, et al. The first draft of the pigeonpea genome sequence. J Plant Biochem Biot. 2012;21(1):98-112.

18. Paterson AH, Wendel JF, Gundlach $H$, Guo H, Jenkins J, Jin DC, Llewellyn D, Showmaker KC, Shu SQ, Udall J, et al. Repeated polyploidization of Gossypium genomes and the evolution of spinnable cotton fibres. Nature. 2012;492(7429):423.

19. Wang KB, Wang ZW, Li FG, Ye WW, Wang JY, Song GL, Yue Z, Cong L, Shang HH, Zhu SL et al. The draft genome of a diploid cotton Gossypium raimondii. Nat Genet 2012, 44(10):1098-+.

20. Li F, Fan G, Wang K, Sun F, Yuan Y, Song G, Ma Z, Lu C, Zou C, et al. Genome sequence of the cultivated cotton Gossypium arboreum. Nat Genet. 2014:46(6):567-72

21. Li F, Fan G, Lu C, Xiao G, Zou C, Kohel RJ, Ma Z, Shang H, Ma X, Wu J, et al. Genome sequence of cultivated Upland cotton (Gossypium hirsutum TM-1) provides insights into genome evolution. Nat Biotechnol. 2015;33(5):524-30.
22. Gu ZL, Cavalcanti A, Chen FC, Bouman P, Li WH. Extent of gene duplication in the genomes of Drosophila, nematode, and yeast. Mol Biol Evol. 2002; 19(3):256-62.

23. Yang $\mathrm{SH}$, Zhang XH, Yue JX, Tian DC, Chen JQ. Recent duplications dominate NBS-encoding gene expansion in two woody species. Mol Gen Genomics. 2008;280(3):187-98.

24. Wang J, Sun N, Deng T, Zhang LD, Zuo KJ. Genome-wide cloning, identification, classification and functional analysis of cotton heat shock transcription factors in cotton (Gossypium hirsutum). BMC Genomics. 2014;15.

25. Schauser L, Wieloch W, Stougaard J. Evolution of NIN-Like proteins in Arabidopsis, rice, and Lotus japonicus. J Mol Evol. 2005:60(2):229-37.

26. Wei KF, Wang YM, Xie DX. Identification and expression profile analysis of the protein kinase gene superfamily in maize development. Mol Breeding 2014;33(1):155-72.

27. Ohno $\mathrm{S}$, Wolf $U$, Atkin NB. Evolution from fish to mammals by gene duplication. Hereditas. 1968;59(1):169-87.

28. Chothia C, Gough J, Vogel C, Teichmann SA. Evolution of the protein repertoire. Science. 2003;300(5626):1701-3.

29. Shih MD, Hsieh TY, Jian WT, Wu MT, Yang SJ, Hoekstra FA, Hsing YIC. Functional studies of soybean (Glycine max L.) seed LEA proteins GmPM6, GmPM11, and GmPM30 by CD and FTIR spectroscopy. Plant Sci. 2012;196: $152-9$.

30. Feng ZH, Zhu J, Du XL, Cui XH. Effects of three auxin-inducible LBD members on lateral root formation in Arabidopsis thaliana. Planta. 2012 236(4):1227-37.

31. Popescu SC, Popescu GV, Bachan S, Zhang ZM, Gerstein M, Snyder M, Dinesh-Kumar SP. MAPK target networks in Arabidopsis thaliana revealed using functional protein microarrays. Gene Dev. 2009;23(1):80-92.

32. Yin ZJ, Wang JJ, Wang DL, Fan WL, Wang S, Ye WW. The MAPKKK gene family in Gossypium raimondii: genome-wide identification, classification and expression analysis. Int J Mol Sci. 2013;14(9):18740-57.

33. Wang YJ, Hao YJ, Zhang ZG, Chen T, Zhang JS, Chen SY. Isolation of trehalose-6-phosphate phosphatase gene from tobacco and its functional analysis in yeast cells. J Plant Physiol. 2005;162(2):215-23.

34. Avonce N, Leyman B, Mascorro-Gallardo JO, Van Dijck P, Thevelein JM, Iturriaga G. The Arabidopsis trehalose-6-P synthase AtTPS1 gene is a regulator of glucose, abscisic acid, and stress signaling. Plant Physiol. 2004: 136(3):3649-59.

35. Tamura K, Stecher G, Peterson D, Filipski A, Kumar S. MEGA6: molecular evolutionary genetics analysis version 6.0. Mol Biol Evol. 2013;30(12):2725-9.

36. Olcott HS. Sand culture of cotton plants. Science. 1939;89(2322):608-9.

\section{Submit your next manuscript to BioMed Central and we will help you at every step:}

- We accept pre-submission inquiries

- Our selector tool helps you to find the most relevant journal

- We provide round the clock customer support

- Convenient online submission

- Thorough peer review

- Inclusion in PubMed and all major indexing services

- Maximum visibility for your research

Submit your manuscript at www.biomedcentral.com/submit 